\title{
MIMO SAR Imaging for Wide-Swath Based on Compressed Sensing
}

\author{
Feng Liu, Shanxiang Mu, and Wanghan Lv \\ School of Electronic and Optical Engineering, Nanjing University of Science and Technology, Nanjing 210094, China \\ Correspondence should be addressed to Feng Liu; liufengnjust@163.com
}

Received 30 August 2016; Revised 31 December 2016; Accepted 19 January 2017; Published 21 February 2017

Academic Editor: Raffaele Solimene

Copyright (C) 2017 Feng Liu et al. This is an open access article distributed under the Creative Commons Attribution License, which permits unrestricted use, distribution, and reproduction in any medium, provided the original work is properly cited.

\begin{abstract}
To reduce the amount of data to be stored and software/hardware complexity and suppress range ambiguity, a novel MIMO SAR imaging based on compressed sensing is proposed under the condition of wide-swath imaging. Random phase orthogonal waveform (RPOW) is designed for MIMO SAR based on compressed sensing (CS). Echo model of sparse array in range and compressive sampling is reconstructed with CS theory. Resolution in range imaging is improved by using the techniques of digital beamforming (DBF) in transmit. Zero-point technique based on CS is proposed with DBF in receive and the range ambiguity is suppressed effectively. Comprehensive numerical simulation examples are performed. Its validity and practicality are validated by simulations.
\end{abstract}

\section{Introduction}

Synthetic aperture radar (SAR) has been widely used in remote sensing imaging technology. However, current singleantenna SAR systems have become more sophisticated and cannot fulfill the increasing demands of future remote sensing in high-resolution and wide-swath (HRWS) imaging performance [1]. On one hand, a small antenna length in azimuth is needed to achieve a fine azimuth resolution. On the other hand, the imaging of large swaths requires low pulse repetition frequencies (PRFs) to avoid range ambiguities, and azimuth ambiguities will appear if the PRF is too low for the chosen azimuth antenna length. In order to suppress range ambiguity, LCMV [2] and MUSIC [3] algorithms of DBF are introduced. So current single-antenna SAR systems cannot provide simultaneously high-resolution and wide-swath imaging. One solution is the displaced phase center antenna (DPCA) technique whose receive antennas are located in along-track direction [4], but the potential drawback is that sampling is nonuniform in azimuth constantly. Another solution is to use multiaperture in receive to gather additional information [5]. The digital beamforming (DBF) on receive techniques is employed.

In recent years, the multiple-input and multiple-output (MIMO) technique is applied to the SAR system of multiple antennas in range $[1,6]$. Orthogonal waveforms are simultaneously transmitted by antennas in range and echoes are isolated from different transmit signals with different weighting coefficients. Using DBF technology resolution in range is improved. The challenge of large amount of data is the common character of the above methods. To solve this problem, compressed sensing technology is used in SAR imaging [7-9]. The effect of sampling rate and the channel capacity on imaging system are analyzed in a given measurement matrix and SNR in [10]. In literature [11], a new CS-SAR imaging method is proposed that can be applied to high-quality and high-resolution imaging under sub-Nyquist rate sampling, while saving the computational cost substantially both in time and in memory.

In addition to solving the problem of large amount of data, waveform design is the key technology to achieve MIMO SAR imaging $[1,12,13]$. Signal waveform should have enough time-bandwidth products and less peak power. According to the requirement of signal waveform, a modified genetic algorithm (GA) is proposed to numerically search optimal frequency firing order for discrete frequency-coding waveform (DFCW) in [14], and linear frequency modulation hybrid coding (LFMHC) waveform is proposed to enable MIMO SAR to operate efficiently in practical applications [15], but the methods can increase the bandwidth of RF 
system. A random OFDM-LFM waveform is proposed in [1] and has good performance in MIMO SAR imaging.

In this paper we proposed a random phase OFDM-LFM waveform whose orthogonality is used to separate the echo signals and beamforming. The proposed waveform has only changed the initial frequency and phase of the LFM signal, so the complexity of the transmitted signal is reduced to meet the requirements of MIMO SAR imaging. Using this waveform, a new MIMO SAR imaging for sparse receive array in range based on CS is proposed. The quantity of receive antennas is reduced whose spacing between the array does not have to satisfy a limit of less than $\lambda / 2$ where $\lambda$ is the transmit wavelength. According to the CS theory, the received echo signals can be directly sampled randomly, and the sampling frequency does not need to meet the Nyquist sampling frequency, which can reduce the sampling data. Zero-point technique based on CS is proposed with DBF in receive to suppress range ambiguity. The rest of this paper is organized as follows. In Section 2, a random phase orthogonal waveform for MIMO SAR is designed. Then compressed sensing theory is introduced in Section 3. In Section 4, illustration and implementation of the signal model of MIMO SAR in range based on CS are proposed. In Section 5, the numerical simulation results are presented. Finally, conclusions are reported in Section 6.

\section{Random Phase Orthogonal Waveform Design}

Waveform design is considered in this section. Waveform design is the key technology to MIMO SAR imaging. Because the linear frequency modulation (LFM) signal has a large time-bandwidth product and it is also the main waveform used by the conventional SAR system, the main research in this paper is based on the LFM signal.

Consider that transmit signals of MIMO SAR are $s(t)=$ $\left[s_{1}(t), s_{2}(t), \ldots, s_{m}(t), \ldots, s_{M}(t)\right]^{T}$, and the $m$ th transmit signal can be expressed by

$$
s_{m}(t)=\operatorname{rect}(t) C_{m} U_{m}(t)
$$

where

$$
\operatorname{rect}(t)= \begin{cases}\frac{1}{\sqrt{T_{P}}}, & 0 \leq t \leq T_{P} \\ 0, & \text { others }\end{cases}
$$

is a rectangular pulse signal, and $C_{m}=\exp \left(j \varphi_{m}\right)$ shows the $m$ th phase encoding signal where $\varphi_{m}=(2 \pi / L) a_{m}$ is random phase, $L$ is the number of available phases for phase encoding, and $a_{m}$ is an element in sequence of $\{0,1, \ldots, L-1\} . U_{m}(t)=$ $\exp \left[j 2 \pi\left(f_{m} t+(1 / 2) K_{m} t^{2}\right)\right]$ is $m$ th LFM signal of random encoding waveform, where $f_{m}$ is the initial frequency of the LFM signal, $K_{m}=b_{m} B / T_{P}$ is the modulation slope, $B$ is pulse signal bandwidth, and $b_{m}$ is a pseudorandom number.

As an example, Figure 1 is a schematic diagram of eight waveforms whose color represents different random phases. Figure 1(a) expresses that the phase and modulation slope are random, and Figures $1(\mathrm{~b})$ and $1(\mathrm{c})$ are the simplified waveforms whose modulation slope is consistent, where modulation slope is up in Figure 1(b) and down in Figure 1(c). In practical applications, the waveforms shown in Figure 1(a) are commonly used in signal processing to matched filter (MF), and the waveforms shown in Figures 1(b) and 1(c) are used for the Stretch processing system. Compared with the matched filter processing, the Stretch processing system only requires one reference signal which uses the waveforms shown in Figure 1(b) or Figure 1(c). The analysis of signal model and simulation mainly uses the waveform shown in Figure 1(b).

Figure 2 is a frequency domain result of eight signals after Stretch processing in one receive channel. The parameters are $B=10 \mathrm{MHz}, T_{p}=10 \mu \mathrm{s}$, the starting frequencies $f_{m}=[50,70,0,20,40,10,60,30] \times 10^{6} \mathrm{~Hz}$, and phase $\varphi_{m}=$ $2 \pi / 8 \times[5,1,4,0,2,3,7,6]$. It can be seen that the echoes are separated after Stretch processing using the random phase orthogonal waveforms. Analyzed by ambiguity function [1, 15], this waveform has a satisfactory ambiguity function performance in range resolution and Doppler frequency resolution.

\section{Compressed Sensing Theory}

Compressed sensing theory is mainly used for sparse signal restoration. As sparse signal representation has greater flexibility in matching structure in the signal, it can be used in SAR imaging. Assume that vector $\mathbf{x}=\left[x_{1}, x_{2}, \ldots, x_{N}\right]^{\mathrm{T}}$ and transform based matrix $\Psi=\left[\psi_{1}, \psi_{2}, \ldots, \psi_{N}\right]$, the signal $\mathbf{x}$ on transform based matrix $\Psi$ can be expressed by

$$
\mathbf{x}=\sum_{n=1}^{N} s_{n} \Psi_{n}=\mathbf{A s}
$$

where $\mathbf{s}=\left[s_{1}, s_{2}, \ldots, s_{N}\right]^{\mathrm{T}}$ is weight coefficient vector of $N \times 1$ dimensions. The signal $\mathbf{x}$ is a $K$-sparse signal if there are $K$ significant coefficients in $\mathbf{S}$ where $K$ is the sparse level, while others are nearly zeros.

Linear measurements of sparse signal $\mathbf{x}$ can be expressed as

$$
\mathbf{y}=\Phi \mathbf{x}
$$

where $\boldsymbol{\Phi}=\left[\begin{array}{llll}\varphi_{1} & \varphi_{2} & \cdots & \varphi_{M}\end{array}\right]$ is measurement matrix.

Put (3) into (4) and we can get

$$
\begin{aligned}
& \mathrm{y}=\Phi_{\mathrm{x}}=\Phi \Psi \mathrm{s}, \\
& \mathrm{y}=\Psi \mathrm{s},
\end{aligned}
$$

where $\mathrm{D}=\boldsymbol{\Phi} \Psi$ is recovery matrix.

In the above transformation process, it is possible to reconstruct the original signal $\mathbf{x}$ from the observation signal $\mathbf{y}$ by the reconstruction algorithm when the restricted isometry property (RIP) is met [16]. Therefore, the design of the measurement matrix $\Phi$ is very important. There are many measurement matrixes that have been used, such as the Hadamard matrix, Gaussian random matrix, sparse random matrix, and part of the Fourier matrix. 


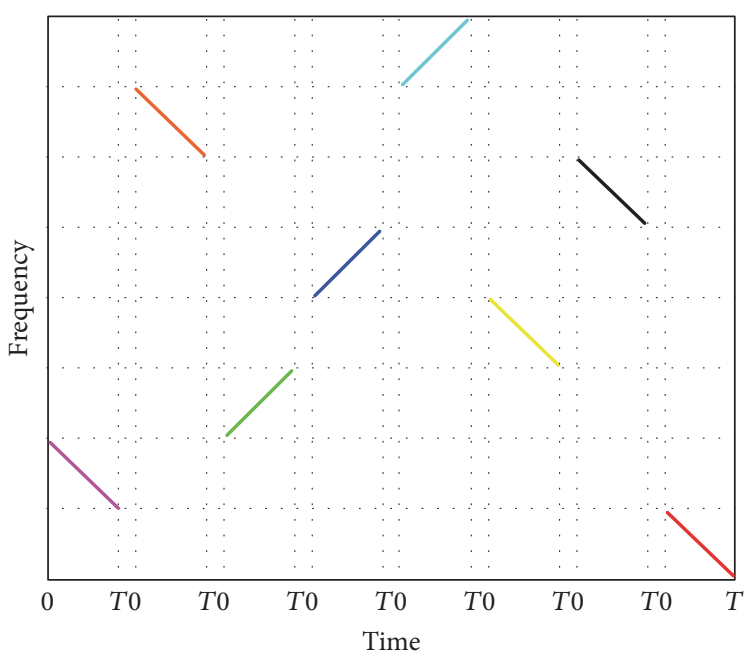

(a)



(b)

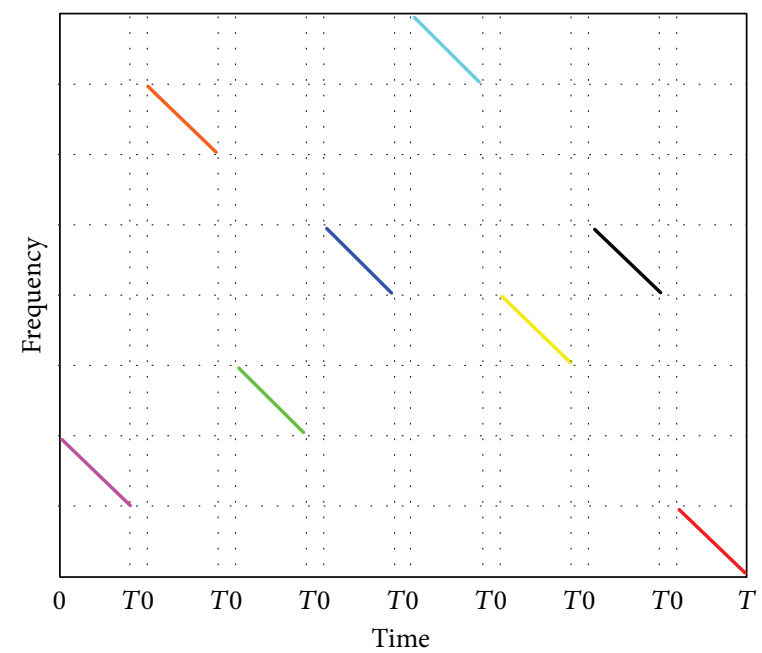

(c)

FIGURE 1: Schematic diagram of random phase orthogonal waveform.

\section{The Signal Model of MIMO SAR in Range Based on CS}

The range ambiguity is existing because MIMO SAR antenna patterns are wider than swath, and the external signals can be received with useful echoes. The echoes of MIMO SAR in range can be used by DBF that the antenna main lobe is pointed to the direction of the desired swath and nulls in the direction of interferers by null steering techniques. Thereby range ambiguity can be suppressed while desired targets can be imaged. Figure 3 is schematic diagram of range ambiguity suppression using null steering techniques.

4.1. Echo Model in One Receive Channel. Echo in one receive channel is the superposition of all reflected signals. Using RPOW signals as shown in Figure 1(b), the $m$ th transmit signal in $n$th receive channel in the receiving array is assumed as

$$
s_{r}=e^{j 2 \pi f_{c}(t-\tau)} e^{j \varphi_{m}} e^{j 2 \pi\left[f_{s m}(t-\tau)+1 / 2 K_{r}(t-\tau)^{2}\right]},
$$

where $f_{s m}$ is starting frequency of random phase orthogonal waveform and $\tau$ is delay time. The expression of the reference signal is

$$
s_{\text {ref }}=e^{j 2 \pi f_{c} t} e^{-j \varphi_{m}} e^{j \pi K_{r} t^{2}} .
$$

The signal of difference frequency mixing after Stretch can be written as

$$
\begin{aligned}
s_{i f} & =s_{r}(t) \cdot s_{\mathrm{ref}}^{*}(t) \\
& =e^{j 2 \pi\left(-f_{c} \tau+(1 / 2) K_{r} \tau^{2}\right)} e^{j 2 \pi f_{s m}(t-\tau)} e^{-j 2 \pi K_{r} \tau t} .
\end{aligned}
$$

Radar echo signal is sparse in Fourier transform domain from (5). Therefore, it can be processed and recovered by compressed sensing theory. Diagram of sparse receive array and signal sampling is shown in Figure 4 , where $N_{r}$ indicates the sampling point in range and $N_{a}$ denotes the sampling point in azimuth. 


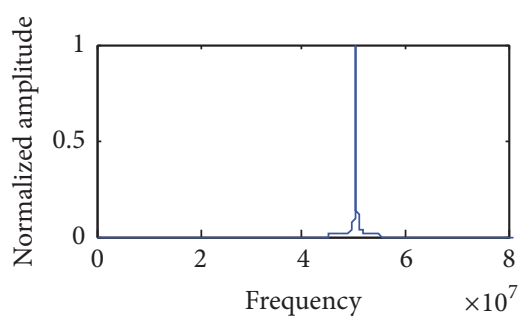

(a)



(d)

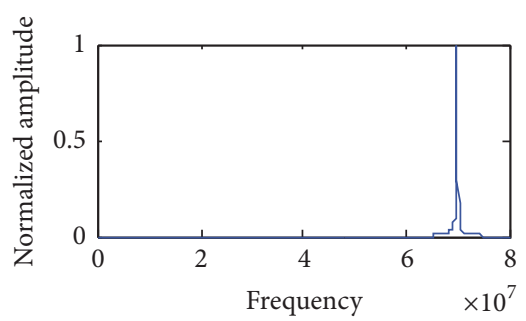

(b)



(e)

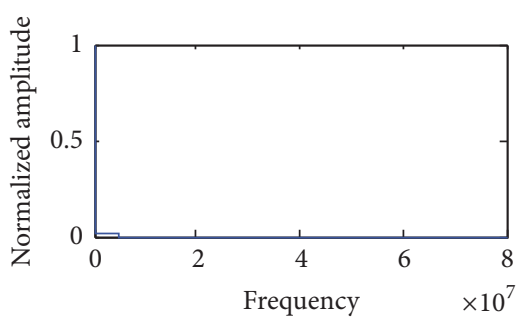

(c)

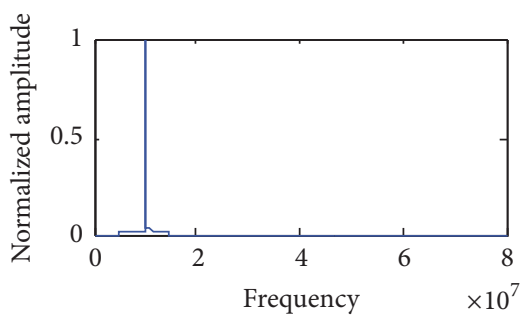

(f)

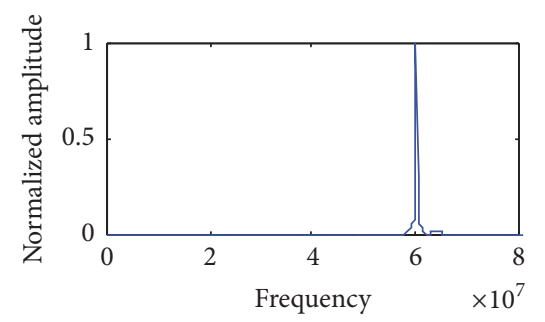

(g)

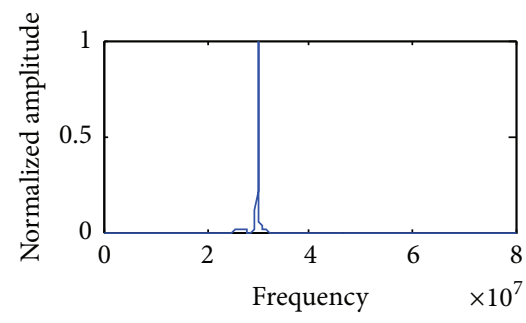

(h)

FIGURE 2: Output of eight signals in frequency domain after Stretch processing in one receive channel.

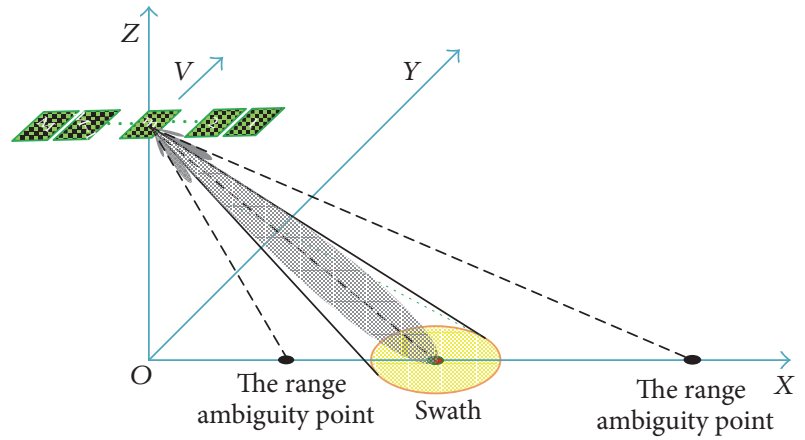

FIGURE 3: Schematic diagram of range ambiguity suppression using null steering techniques.

When the sample number $n_{c}$ is large enough, after discretization, a band limited analog signal can be recovered by

$$
x_{r}(t)=\sum_{n_{c}=-N_{c} / 2}^{N_{c} / 2-1} x\left[n_{c}\right] \frac{\sin \left[\pi\left(t-n_{c} T\right) / T\right]}{\pi\left(t-n_{c} T\right) / T},
$$

where $x\left[n_{c}\right]$ is sample sequence of original signal $x_{s}$ which meets the Nyquist frequency and $T$ is sampling period.

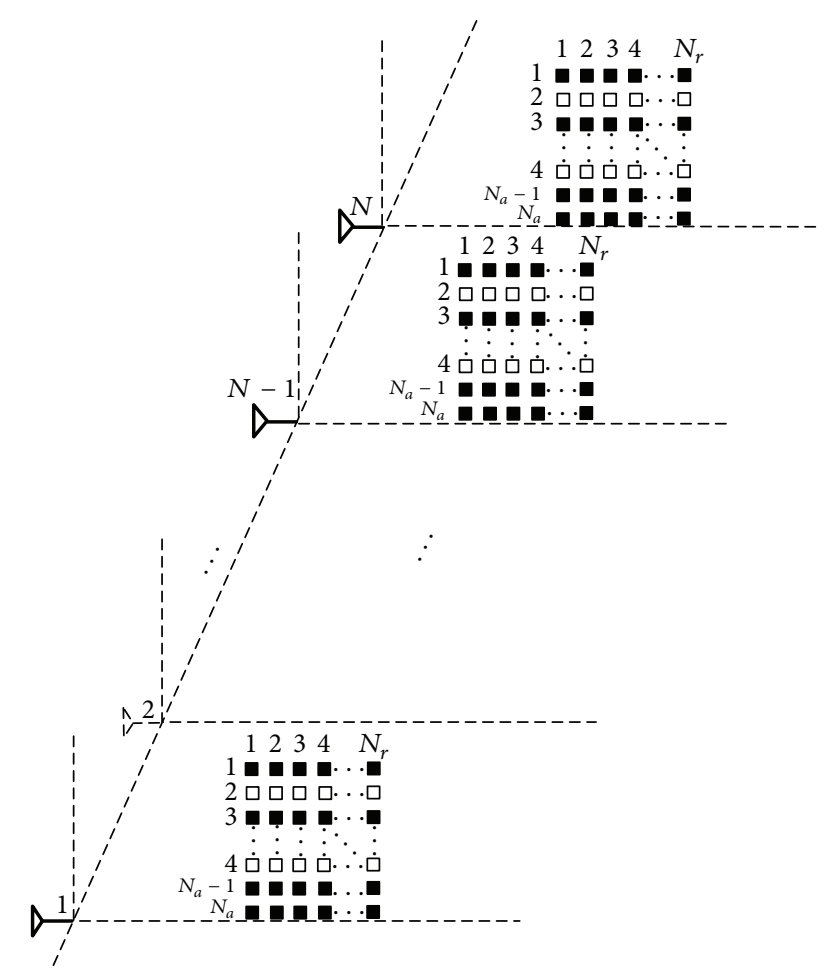

FIGURE 4: Diagram of sparse receive array and signal sampling. 


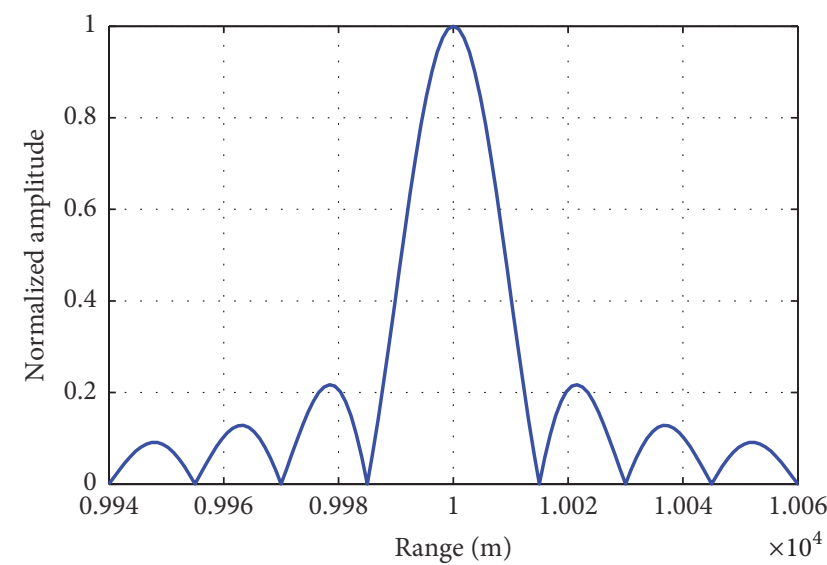

(a) Output of single LFM

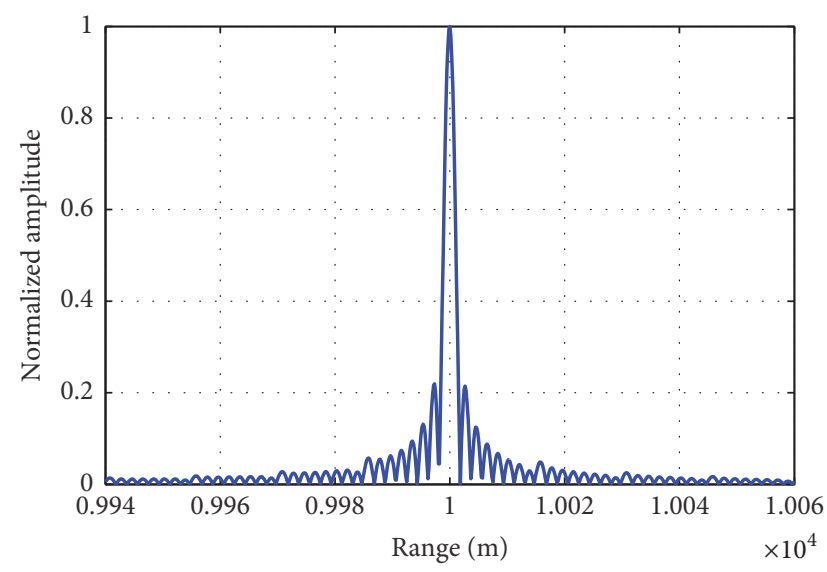

(c) The final output of RPOW signal

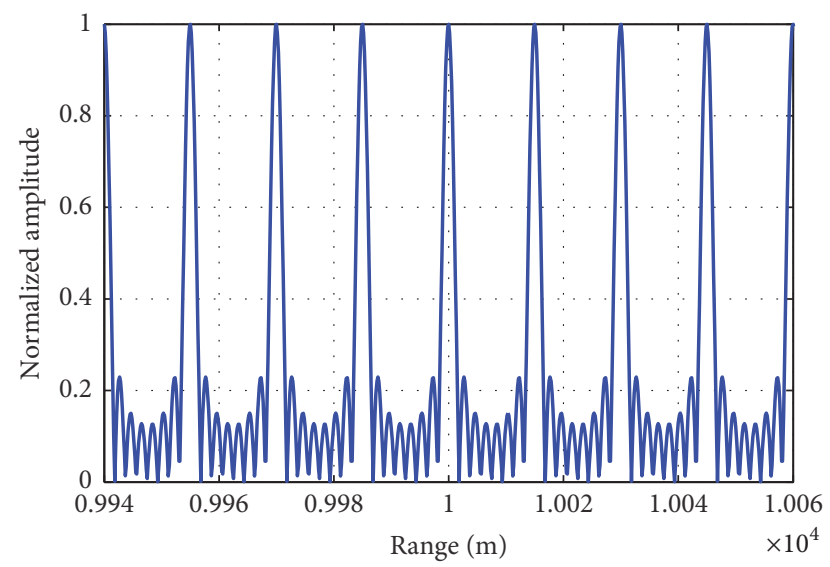

(b) Output of RPOW signal with DBF

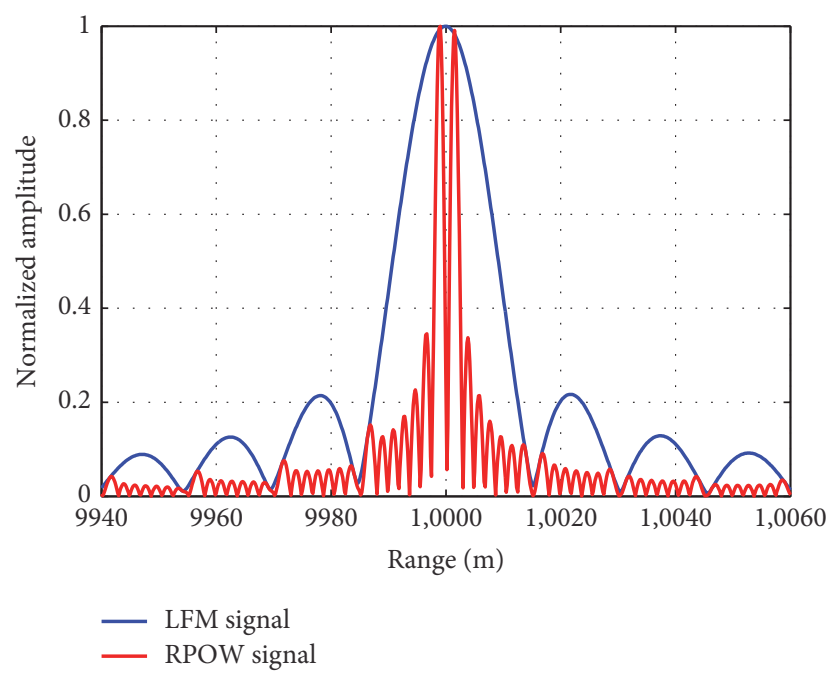

(d) Resolution comparison of single LFM and RPOW signal

FIGURE 5: Resolution comparison of single LFM signal with RPOW signal.

The original signal is restored from $M_{c}$ random samples of $N_{c}$ samples. Sampled sequence $s\left(\Delta t_{m}\right)$ can be expressed by

$$
s\left(\Delta t_{m}\right)=\sum_{n_{c}=1}^{N_{c}} x\left(n_{c} T_{e}\right) \frac{\sin \left[\pi\left(\Delta t_{m}-n_{c} T_{e}\right) / T_{e}\right]}{\pi\left(\Delta t_{m}-n_{c} T_{e}\right) / T_{e}},
$$

where $\Delta t_{m}$ is relative time interval of $m$ th random sample, $1 \leq m \leq M_{c}$, and $T_{e}$ is random sampling interval which can be considered the same as $T$.

The relationship between the random sample values and sample values satisfying the Nyquist sampling rate can be expressed as [17]

$$
\left[\begin{array}{c}
s\left(\Delta t_{1}\right) \\
s\left(\Delta t_{2}\right) \\
\vdots \\
s\left(\Delta t_{M}\right)
\end{array}\right]=\left[\begin{array}{cccc}
\phi_{11} & \phi_{12} & \cdots & \phi_{1 N_{c}} \\
\phi_{21} & \phi_{22} & \cdots & \phi_{2 N_{c}} \\
\vdots & \vdots & \ddots & \vdots \\
\phi_{M 1} & \phi_{M 2} & \cdots & \phi_{M_{c} N_{c}}
\end{array}\right]\left[\begin{array}{c}
s\left(T_{e}\right) \\
s\left(2 T_{e}\right) \\
\vdots \\
s\left(N_{c} T_{e}\right)
\end{array}\right] .
$$

Using vector representation we have

$$
\mathbf{s}=\boldsymbol{\Phi} \mathbf{x},
$$

where $\boldsymbol{\Phi}$ is measure matrix and $\phi_{m_{c} n_{c}}=\sin \left[\pi\left(\Delta t_{m}-n_{c} T_{e}\right) /\right.$ $\left.T_{e}\right] /\left(\pi\left(\Delta t_{m}-n_{c} T_{e}\right) / T_{e}\right)$.

Transformation matrix is

$$
\Psi=\left[\begin{array}{cccc}
1 & e^{-i \Delta \phi_{B 1}} & e^{-i 2 \Delta \phi_{B 1}} & e^{-i(N-1) \Delta \phi_{B 1}} \\
1 & e^{-i \Delta \phi_{B 2}} & e^{-i 2 \Delta \phi_{B 2}} & e^{-i(N-1) \Delta \phi_{B 2}} \\
\vdots & \vdots & \ddots & \vdots \\
1 & e^{-i \Delta \phi_{B M}} & \cdots & e^{-i(N-1) \Delta \phi_{B M}}
\end{array}\right] .
$$

Multibeam can also be restored by this transformation matrix in DBF.

To solve the problem of sparse signal representation, various methods have been proposed, such as basis pursuit $[18,19]$ and orthogonal matching pursuit $[20,21]$. This paper takes orthogonal matching pursuit (OMP) algorithm to recover the original signal. 

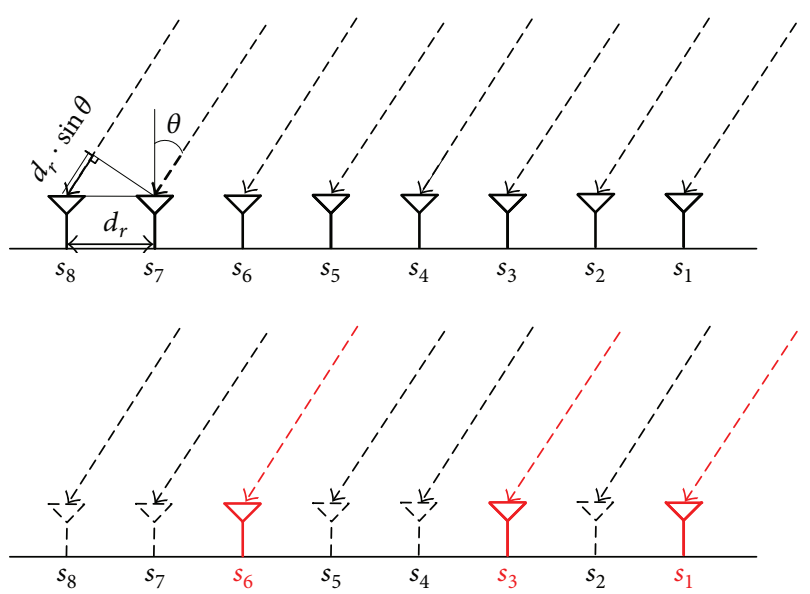

FIGURE 6: Schematic diagram of sparse array in receive.

4.2. DBF in Transmit. Suppose that the transmit array is a uniform linear array, the transmit signals can be expressed by

$$
\mathbf{s}_{t}(t)=\mathbf{v}_{t}^{\mathrm{T}}\left(\theta_{t}\right)\left[s_{1}(t), s_{2}(t), \ldots, s_{M}(t)\right]^{\mathrm{T}}
$$

where $\mathbf{v}_{t}^{\mathrm{T}}\left(\theta_{t}\right)=\left[1, e^{-j(2 \pi / \lambda) d_{0} \sin \theta_{t}}, \ldots, e^{-j(2 \pi / \lambda)(M-1) d_{0} \sin \theta_{t}}\right]^{\mathrm{T}}$ is transmit array response vector and $\theta_{t}$ is the incidence angle in the transmit array response vector. The receive signals can be written as

$$
\begin{aligned}
\mathbf{x}_{r}(t) & =\mathbf{v}_{r}\left(\theta_{r}\right) \mathbf{s}_{t}\left(t-\tau_{d}\right)=\mathbf{v}_{r}\left(\theta_{r}\right) \mathbf{v}_{t}^{\mathrm{T}}\left(\theta_{t}\right) \\
\cdot & {\left[s_{1}\left(t-\tau_{d}\right), s_{2}\left(t-\tau_{d}\right), \ldots, s_{M}\left(t-\tau_{d}\right)\right]^{\mathrm{T}}, }
\end{aligned}
$$

where $\mathbf{v}_{r}\left(\theta_{r}\right)=\left[1, e^{-j(2 \pi / \lambda) d_{0} \sin \theta_{r}}, \ldots, e^{-j(2 \pi / \lambda)(N-1) d_{0} \sin \theta_{r}}\right]^{\mathrm{T}}$ is the receive array response vector and $\tau_{d}$ represents the time which the signal takes to travel the transmitter-target-receiver distance.

With Stretch process and band pass filter, the echo expressed in (9) can be performed with DBF. With DBF in $n$th receive channel, the output can be expressed by

$$
\begin{gathered}
F_{B}(b)=\sum_{m=0}^{M-1} e^{j\left(2 \pi d_{0} / \lambda\right)(m-1) \sin \left(\phi_{b}\right)} e^{-j\left(2 \pi d_{0} / \lambda\right)(m-1) \sin \left(\theta_{t}\right)} \\
\cdot e^{j 2 \pi\left[f_{s m}(t-\tau)-f_{c} \tau\right]} e^{-j 2 \pi K_{r} \tau t} e^{-j(2 \pi / \lambda)(n-1) d_{0} \sin \theta_{r}},
\end{gathered}
$$

where $\phi_{b}$ is beam direction. When $\phi_{b}=\theta_{t}$, the output can be expressed as

$$
\begin{aligned}
& F_{B \theta_{t}}(b) \\
& =\sum_{m=0}^{M-1} e^{-j 2 \pi f_{c} \tau} e^{j 2 \pi f_{s m}(t-\tau)} e^{-j 2 \pi K_{r} \tau t} e^{-j(2 \pi / \lambda)(n-1) d_{0} \sin \theta_{r}} \\
& =e^{-j 2 \pi f_{c} \tau} e^{-j 2 \pi K_{r} \tau t} e^{-j(2 \pi / \lambda)(n-1) d_{0} \sin \theta_{r}} \sum_{m=0}^{M-1} e^{j 2 \pi f_{s m}(t-\tau)} .
\end{aligned}
$$

Now we consider the expression (9). Each received signal is processed by demodulation. The expression in frequency domain is

$$
\begin{aligned}
S_{i f_{s n} c r n} & (f) \\
= & e^{-j\left(2 \pi d_{0} / \lambda\right)(m-1) \sin \left(\theta_{t}\right)} \\
& \cdot \sin c\left[T_{p} 2 \pi\left(f+K_{r} \tau\right)\right] e^{-j 2 \pi f_{c} \tau} e^{-j 2 \pi f_{s m} \tau} .
\end{aligned}
$$

Because of the correspondence between the frequency and time, the expression (19) can be written as

$$
\begin{aligned}
\left|s_{i f_{s n} c r n}(t)\right| & =\left|S_{i f_{s n} c r n}\left(\frac{f}{K_{r}}\right)\right| \\
& =\left|\sin c\left[\frac{T_{p} 2 \pi\left(f+K_{r} \tau\right)}{K_{r}}\right]\right| \\
& =\left|\sin c\left[T_{p} 2 \pi(t+\tau)\right]\right| .
\end{aligned}
$$

After symmetric processing of $y$-axis, we can get

$$
\text { fliplr }\left(\left|s_{i f_{s n} c r n}(t)\right|\right)=\left|\sin c\left[T_{p} 2 \pi(t-\tau)\right]\right| .
$$

Multiplying the formulae (18) and (21), the new expression is

$$
\begin{aligned}
& H F_{B \theta_{t}}(b)=F_{B \theta_{t}}(b) \cdot \text { fliplr }\left(\left|s_{i f_{s n} c r n}(t)\right|\right) \\
& =e^{-j 2 \pi f_{c} \tau} e^{-j 2 \pi K_{r} \tau t} e^{j((M-1) / 2) f_{s}(t-\tau)} e^{-j(2 \pi / \lambda)(n-1) d_{0} \sin \theta_{r}} \\
& \quad \cdot \frac{\sin \left((M / 2) f_{s}(t-\tau)\right)}{\sin \left((1 / 2) f_{s}(t-\tau)\right)} \cdot\left|\sin c\left[T_{p} 2 \pi(t-\tau)\right]\right|,
\end{aligned}
$$

where

$$
\tau=\frac{2 R\left(t_{\text {slow }}, R_{0}\right)}{C} \approx \frac{2}{C}\left[R_{0}+\frac{\left(V t_{\text {slow }}\right)^{2}}{2 R_{0}}\right]
$$

and $R_{0}$ is the closest slant range to the target.

Considering the envelope, the second addend in (23) can be ignored; that is, $\tau \approx 2 R_{0} / C$, and the expression (22) is written as

$$
\begin{aligned}
& H F_{B \phi_{t}}(b) \\
& =e^{-j(2 \pi / \lambda)(n-1) d_{0} \sin \theta_{r}} e^{-j 2 \pi\left(f_{c} \tau+K_{r} \tau t\right)} e^{j((M-1) / 2) f_{s}(t-\tau)} \\
& \quad \cdot \frac{\sin \left((N / 2) f_{s}\left(t-2 R_{0} / C\right)\right)}{\sin \left((1 / 2) f_{s}\left(t-2 R_{0} / C\right)\right)} \\
& \quad \cdot\left|\sin c\left[T_{p} 2 \pi\left(t-\frac{2 R_{0}}{C}\right)\right]\right| .
\end{aligned}
$$

Taking the absolute value of (24), the expression is

$$
\begin{aligned}
\left|H F_{B \phi_{t}}(b)\right|= & \left|\frac{\sin \left((M / 2) f_{s}\left(t-2 R_{0} / C\right)\right)}{\sin \left((1 / 2) f_{s}\left(t-2 R_{0} / C\right)\right)}\right| \\
& \cdot\left|\sin c\left[T_{p} 2 \pi\left(t-\frac{2 R_{0}}{C}\right)\right]\right| .
\end{aligned}
$$




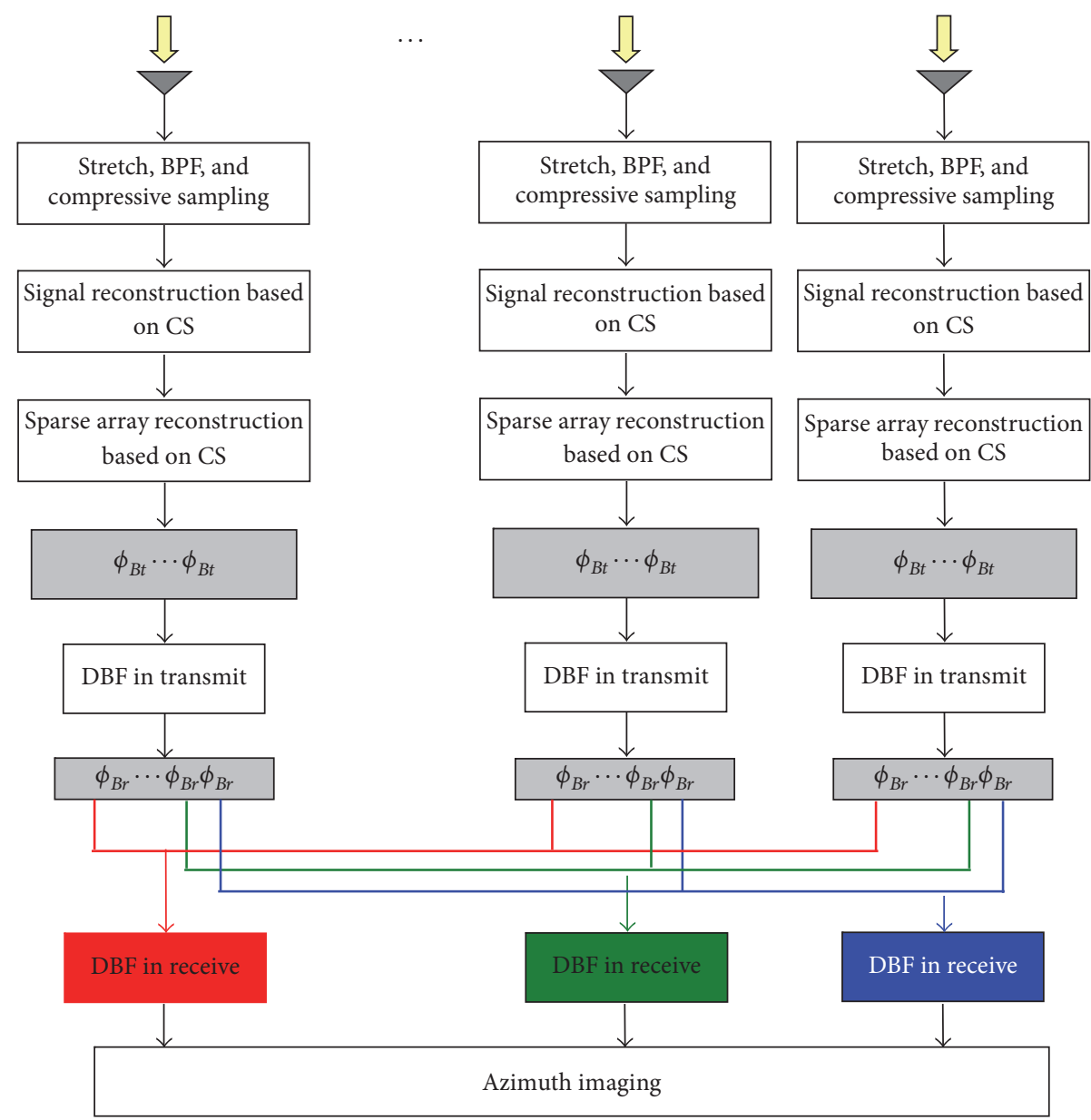

FIGURE 7: MIMO SAR imaging process based on CS.

Envelope of the first factor in right side of (25) is $\sin c$ function shape, and the $-4 \mathrm{~dB}$ width is $\Delta R_{1}=C /\left(2 M f_{s}\right)$. The total resolution after the product is $\Delta R_{\text {total }} \approx \Delta R_{1}=$ $C /(2 M B)$. Thus, resolution of random phase orthogonal waveform is $M$ times resolution of single LFM signal where $M$ is the number of transmit waveforms.

Figure 5 is a schematic diagram of output with RPOW signal. The number of transmit channels is eight, and the target range is set to $10000 \mathrm{~m}$. Figure 5(a) shows the resolution of single LFM signal with Stretch processing. Figure 5(b) is output of RPOW signal in one receiving channel after the digital beamforming which is expressed in (18), and Figure $5(\mathrm{c})$ is waveform processed by multiplying which is expressed in (25). So resolution is improved by eight times which is equal to the number of transmit signals. Figure 5(d) represents output comparison of single LFM signal with random phase orthogonal waveform. Two targets are set to $9999 \mathrm{~m}$ and $10001 \mathrm{~m}$. As shown in Figure 5(d), the two targets are distinguished easily with random phase orthogonal waveform.

4.3. Echo Model of Random Sparse Array. Figure 6 shows the uniform linear array where the number of receive array elements is $N=8$. The array space arrangements satisfy $d_{0} \leq$ $\lambda / 2$. In the figure below shown in Figure 6, the red elements indicate the randomly selected 3 elements, whose numbers are 1,3 , and 6 , respectively. The incident angle of target signal $u(t)$ is $\theta$. The phase difference in space is $\Delta \phi=(2 \pi / \lambda) d_{0} \sin \theta$ and the phase difference in array is $\Delta \phi_{B}=(2 \pi / \lambda) d_{0} \sin \phi_{B}$. The $k$ th beam-pointing is $\phi_{B k}$, and the phase compensation value provided by the digital beamforming processor shall be $\Delta \phi_{B k}=(2 \pi / \lambda) d_{0} \sin \phi_{B k}$. The antenna pattern function of uniform linear array can be expressed as

$$
F(k)=\sum_{i=0}^{N-1} e^{j i\left(\Delta \phi-\Delta \phi_{B k}\right)} .
$$

For receiving $\mathrm{DBF}$, assume that the direction of arrival of the desired signal is $\theta_{1}$, and the arrival directions of the interferences are $\theta_{2}, \ldots, \theta_{k}, \ldots, \theta_{K}$. The steering vector of the receive signals can be expressed as

$$
\mathbf{a}\left(\sin \theta_{k}\right)=\left(1, e^{j 2 \pi d \sin \theta_{k} / \lambda}, \ldots, e^{j 2 \pi(N-1) d \sin \theta_{k} / \lambda}\right)^{\mathrm{T}} .
$$

The echo signal of receive array can be represented as a matrix by

$$
\mathbf{s}(t)=\sum_{k=1}^{K} u_{k}(t) \mathbf{a}\left(\sin \theta_{k}\right)
$$







(a)

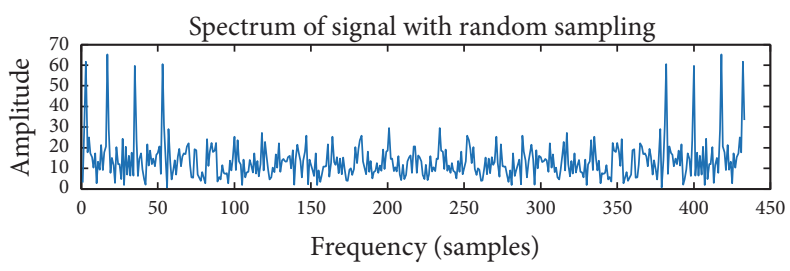

(b)
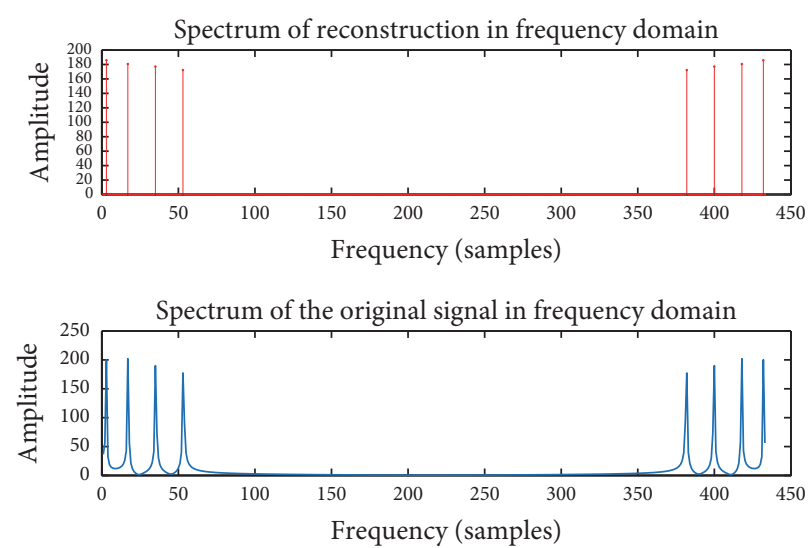

(c)

FIGURE 8: Simulation results of image in range based on CS. (a) shows the original echo of four targets and the echo with compressive sampling of one transmit signal. (b) shows the spectrum of original echo and the echo with compressive sampling, and (c) shows the spectrum of reconstruction with CS method.

In the real scene, echoes can be from multiple directions in receiving window because of range ambiguity. In order to estimate direction of arrival, we decompose the entire space from $-90^{\circ}$ to $90^{\circ}$ into $\Gamma$ parts; then we obtain transformation matrix:

$$
\mathbf{A}=\left[\mathbf{a}\left(\sin \theta_{1}\right), \mathbf{a}\left(\sin \theta_{2}\right), \ldots, \mathbf{a}\left(\sin \theta_{\Gamma}\right)\right]
$$

The echo signal of the receiving array can be expressed as

$$
\mathbf{S}(t)=\mathbf{A U}(t)
$$

where $\mathbf{U}(t)=\left[0,0, \ldots, u_{1}(t), 0, \ldots, 0, \ldots, u_{K}(t), 0, \ldots, 0\right]^{\mathrm{T}}$. It is clear that $\mathbf{U}(t)$ is sparse and has a few nonzero elements. Therefore, according to CS theory, the received signal $\mathbf{S}(t)$ can be recovered accurately by using a CS reconstruction algorithm.

The compressed vector $\mathbf{Y}(t)$ can be expressed with measurement matrix $\Phi$ as

$$
\mathbf{Y}(t)=\boldsymbol{\Phi S}(t)=\boldsymbol{\Phi} \mathbf{A U}(t)=\Psi \mathbf{U}(t) .
$$

Therefore, the reconstruction of the received signal becomes an optimal estimation of (32)

$$
\begin{array}{ll}
\min & \|\widehat{\mathbf{U}}\|_{0} \\
\text { s.t. } & \mathbf{Y}=\Psi \widehat{\Psi} .
\end{array}
$$

After the projection coefficient vector $\widehat{\mathbf{U}}$ is estimated from the compressed vector $\mathbf{Y}(t)$, the received signal $\widehat{\mathbf{S}}(t)$ can be reconstructed, as shown in the following equation:

$$
\widehat{\mathbf{S}}(t)=\mathbf{A} \widehat{\mathbf{U}}(t) \text {. }
$$

The signal can be reconstructed using orthogonal matching pursuit algorithm in this paper.

After obtaining the reconstructed echoes using compressed sensing reconstruction methods, using LCMV beamforming algorithm $[2,22]$, the adaptive digital beamforming is implemented that the antenna main lobe is pointed to the direction of the desired signal and nulls in the direction of interferers. Thereby range ambiguity can be suppressed while desired targets can be imaged.

4.4. MIMO SAR Imaging Process Based on CS. In order to reduce the amount of data, the received signal of Stretch 

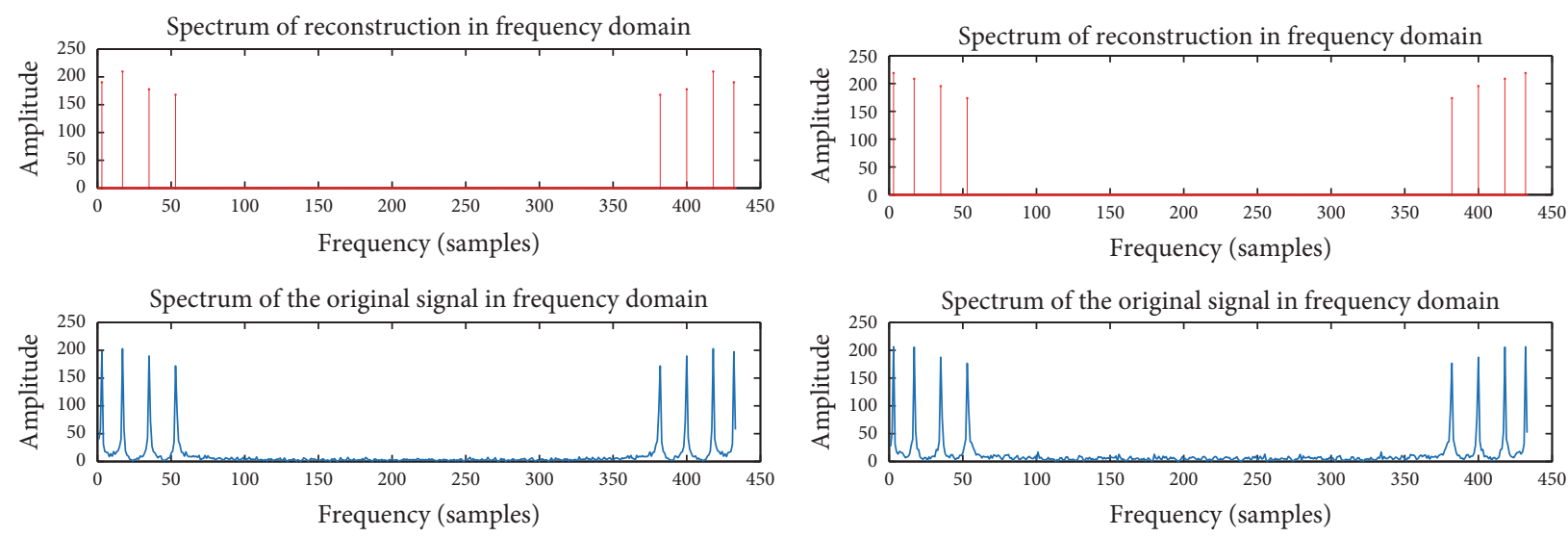

(a) $\mathrm{SNR}=20 \mathrm{~dB}$

(b) $\mathrm{SNR}=15 \mathrm{~dB}$
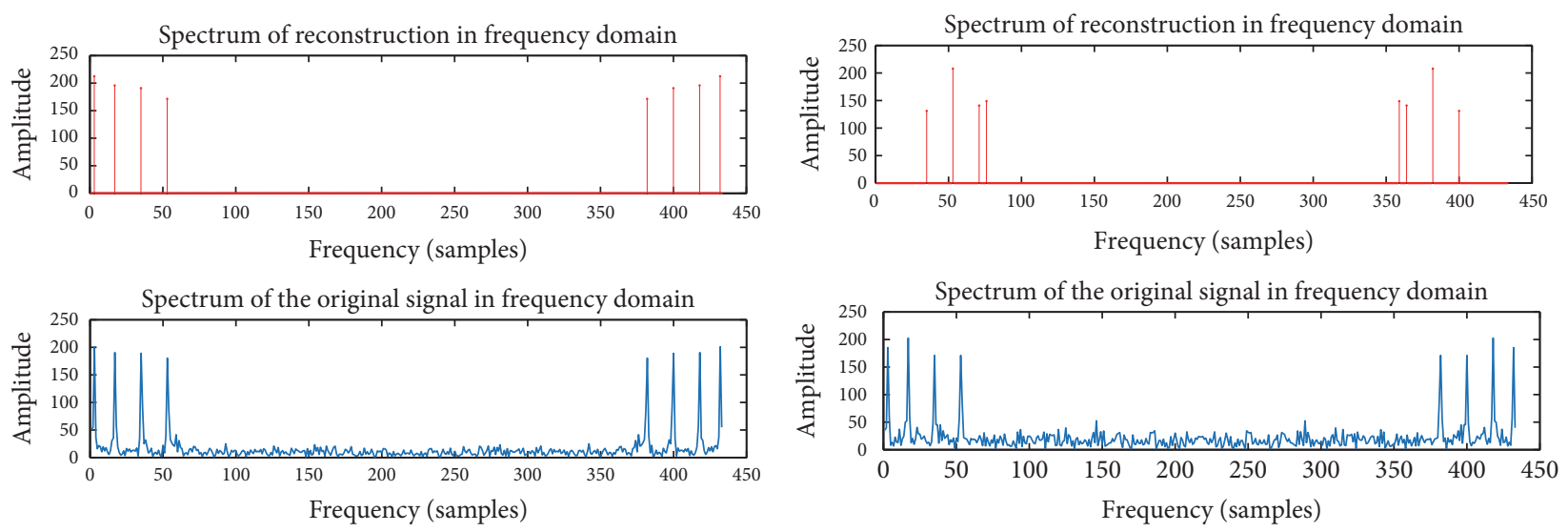

(c) $\mathrm{SNR}=10 \mathrm{~dB}$

(d) $\mathrm{SNR}=5 \mathrm{~dB}$

FIgURE 9: The spectrum of reconstruction in different SNR. (a) shows that the reconstruction in the case of $\mathrm{SNR}=20 \mathrm{~dB}$, (b) shows $\mathrm{SNR}=$ $15 \mathrm{~dB}$, (c) shows $\mathrm{SNR}=10 \mathrm{~dB}$, and (d) shows $\mathrm{SNR}=5 \mathrm{~dB}$.

process is compressive sampled from the random equivalent sampling method and is restored with CS. Then DBF is implemented in transmit in receive channel. So the range resolution can be improved by $M$ times. With DBF in transmit of sparse array, the null steering techniques based on CS is put forward to suppress echo outside of swath in order to range ambiguity suppression. Under normal circumstances, the impact of adjacent ambiguity region with imaging swath is considered because the ambiguity echo power in adjacent ambiguity region generally accounts for more than $80 \%$ of all ambiguity echo power. For the far ambiguity region, the antenna sidelobe gain is low and the echo power is less due to the far distance. MIMO SAR imaging process based on CS is shown in Figure 7.

\section{Simulation Analysis}

5.1. Signal Recovery from the Random Sampling Method in Range Based on CS. In this subsection, some simulation results under different situations are provided. Assume that there are four targets, and the parameters of four RPOW signals are as follows: the starting frequencies $f_{s m}=$ $[0,20,10,30] \times 10^{6} \mathrm{~Hz}, B=10 \mathrm{MHz}, T_{p}=10 \mu \mathrm{s}$, and sparse ratio is 0.37 . Figure 8 shows the simulation results.
It is shown that the amount of data is significantly reduced with compressive sampling, and the frequency spectrum of the targets can be displayed correctly after the restoration compared with the original signal.

Secondly, the influence of SNR is evaluated with CS method. The parameters are the same as in Figure 8. Figure 9 shows the spectrum of reconstruction compared with the original signal. It is shown that the spectrum of reconstruction can display correctly the targets when SNR $>10 \mathrm{~dB}$, but when SNR $<10 \mathrm{~dB}$, such as SNR $=5 \mathrm{~dB}$, the spectrum of reconstruction shows that the target will be lost or false targets appear.

Now let us consider the influence of transmit number. Figure 10 shows the reconstruction spectrum of the RPOW signals from five to eight. The starting frequencies are randomly chosen from $[0,10,20,30,40,50,60,70] \times 10^{6} \mathrm{~Hz}$. Other parameters are as the same as in Figure 7. The recovered signal can display the position of the spectrum correctly, which shows that the increase of the number of signals will not affect the recovery of the data.

Then we consider the Monte Carlo simulation. SNR and sparse ratio are two important indexes in the data recovery of MIMO SAR based on compressed sensing. When SNR or sparse ratio is low, the original signal may not be able 



(a) $M=5$
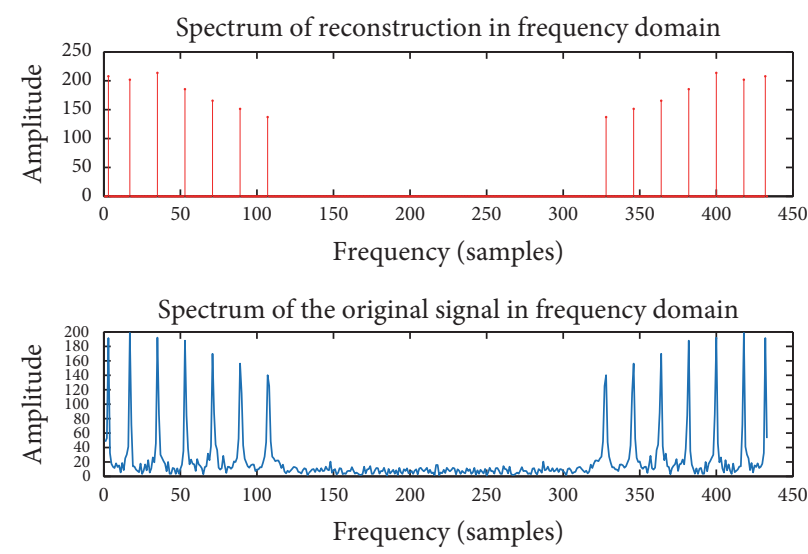

(c) $M=7$
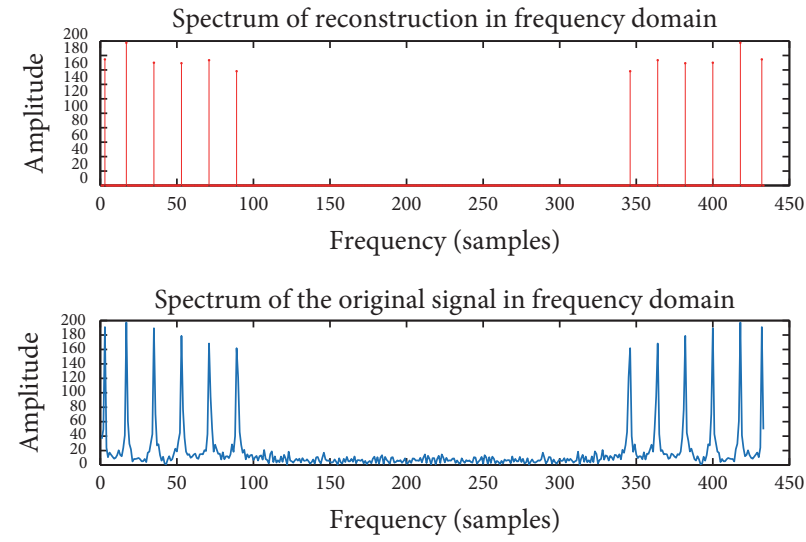

(b) $M=6$
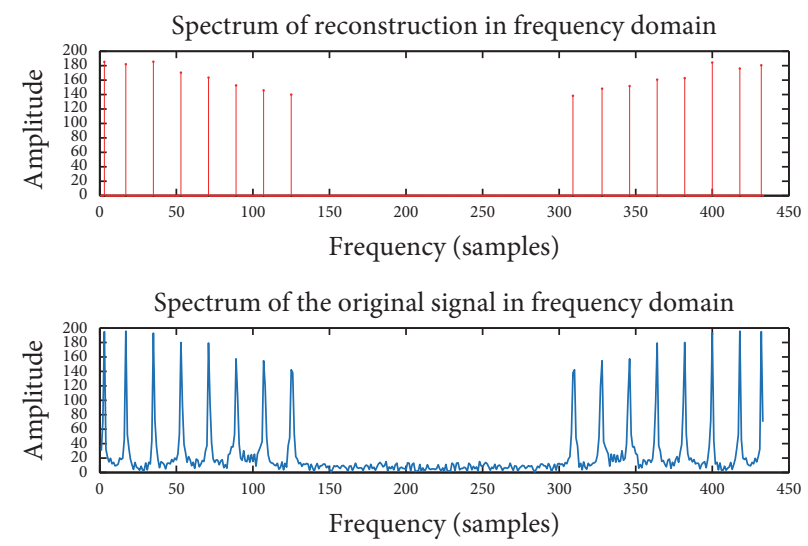

(d) $M=8$

Figure 10: The reconstruction spectrum of different numbers of RPOW signals. (a) shows that the number is 5, (b) shows 6, (c) shows 7, and (d) shows 8 .

to recover correctly. In order to analyze the application of MIMO SAR data recovery based on compressed sensing, we give the following simulation analysis. The carrier frequency is $1 \mathrm{GHz}, f_{s m}=[0,20,10,30] \times 10^{6} \mathrm{~Hz}, B=10 \mathrm{MHz}, T_{p}=$ $10 \mu \mathrm{s}, \mathrm{SNR}$ is from $-5 \mathrm{~dB}$ to $20 \mathrm{~dB}$, and sparse ratio is from 0.2 to 1 . We do Monte Carlo analysis 200 times and give the results of successful probability in Figure 11. It can be seen that successful probability of reconstruction is more than $95 \%$ when the sparse ratio is 0.4 and SNR is greater than $10 \mathrm{~dB}$. At the same SNR, the higher the sparse ratio, the more successful the probability. Under the condition of the same sparse ratio, the higher the SNR, the more successful the probability. Due to the fact that random sampling requires the signal above the noise level, and in the case of small signal, the sparse coefficient is submerged in the noise, so it cannot be well estimated. Small signal will be lost and the probability of success is reduced. Therefore, this method is not suitable for small signal.

5.2. DBF Algorithm of Zero-Pointing Technology Based on CS. To verify the correctness of the algorithm in the cases of sparse array, we choose 30 elements sparsely from 100 elements. Set SNR $5 \mathrm{~dB}$ and $10 \mathrm{~dB}$, INR $10 \mathrm{~dB}$ and $40 \mathrm{~dB}$, and the directions of range ambiguity are set to $-10^{\circ}$ and $10^{\circ}$.
The angular space within the swath is discretized into 720 subspaces. Figure 12 shows the beam patterns with LCMV algorithm in different SNR and INR. It is shown that when SNR is lower than $10 \mathrm{~dB}$, the interference can be suppressed, and the greater the interference, the greater the depth. But the precision is not ideal. When the SNR is greater than $10 \mathrm{~dB}$, and the number of array elements is reduced from 100 to 30 , the data recovery after beamforming, the performance of the proposed algorithm is almost the same as that of the full array. The main reason is that, in the case of low SNR, the signal is relatively low to noise level, and the signal cannot be successfully restored.

5.3. MIMO SAR Imaging Simulation Based on CS. Imaging simulations are also performed to evaluate the algorithm based on CS. Figure 13 shows imaging of HRWS SAR and MIMO SAR based on CS. The parameters are as follows: carrier frequency $f_{c}=10 \mathrm{GHz}$, platform altitude is $600 \mathrm{~km}$, platform velocity is $7640 \mathrm{~m} / \mathrm{s}$, pulse repeated frequency is $1908 \mathrm{~Hz}$, minimum slant range is $558 \mathrm{~km}$, maximum slant range is $727 \mathrm{~km}$, and $\mathrm{SNR}=10 \mathrm{~dB}$, and we apply RPOW waveform to MIMO SAR as shown in Figure 1(b). In order to reduce the computational complexity, number of subswathes is set to 2 , and there are two point targets locating at the 


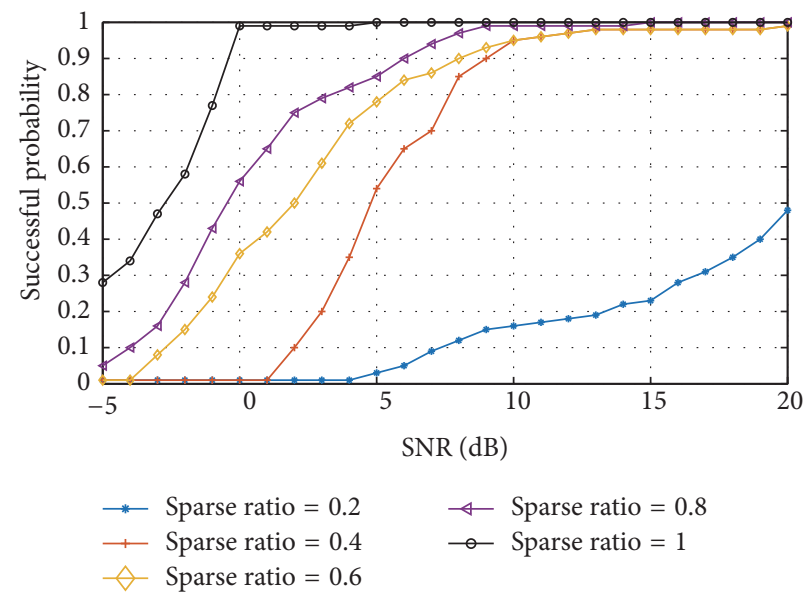

FIGURE 11: Successful probability with different SNR and sparse ratio.

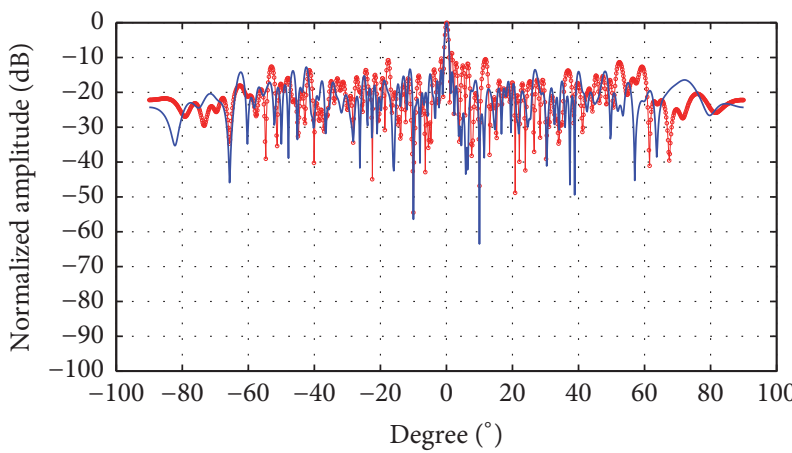

Algorithm based on CS of 30 elements

— Full array with 100 elements

(a) $\mathrm{SNR}=5 \mathrm{~dB}, \mathrm{INR}=10 \mathrm{~dB}$

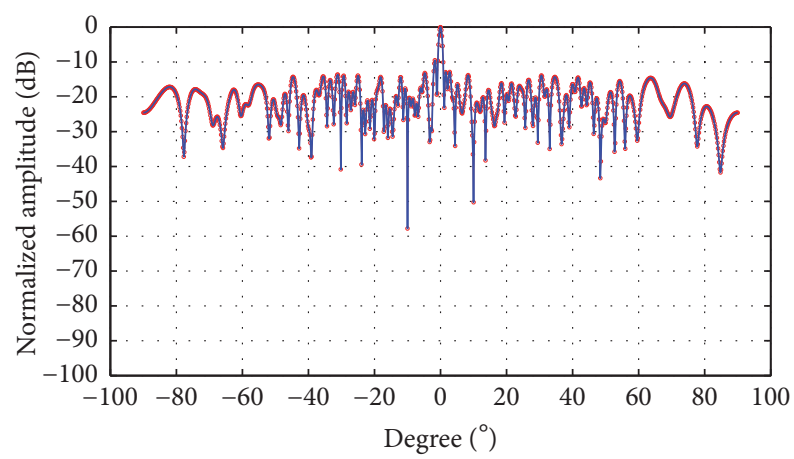

- Algorithm based on CS of 30 elements

— Full array with 100 elements

(c) $\mathrm{SNR}=10 \mathrm{~dB}, \mathrm{INR}=10 \mathrm{~dB}$

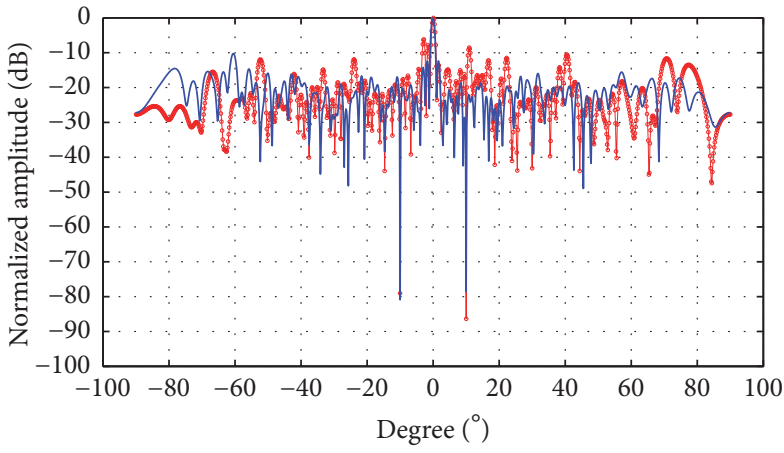

- Algorithm based on CS of 30 elements

— Full array with 100 elements

(b) $\mathrm{SNR}=5 \mathrm{~dB}, \mathrm{INR}=40 \mathrm{~dB}$

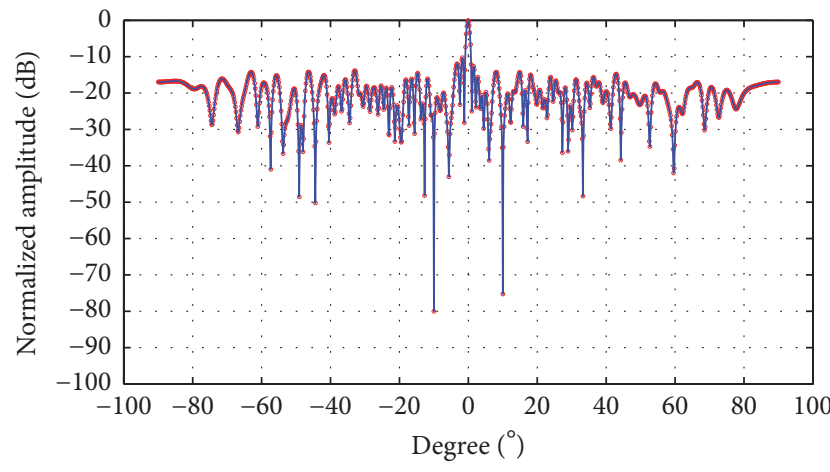

- Algorithm based on CS of 30 elements

— Full array with 100 elements

(d) $\mathrm{SNR}=10 \mathrm{~dB}, \mathrm{INR}=40 \mathrm{~dB}$

FIGURE 12: The beam patterns with different SNR and INR. (a) shows reconstruction in the case of $\mathrm{SNR}=5 \mathrm{~dB}, \mathrm{INR}=10 \mathrm{~dB}$, (b) shows SNR $=5 \mathrm{~dB}, \mathrm{INR}=40 \mathrm{~dB},(\mathrm{c})$ shows $\mathrm{SNR}=10 \mathrm{~dB}, \mathrm{INR}=10 \mathrm{~dB}$, and $(\mathrm{d})$ shows $\mathrm{SNR}=10 \mathrm{~dB}, \mathrm{INR}=40 \mathrm{~dB}$.

positions of $(600 \mathrm{~km}, 0)$ and $(685 \mathrm{~km}, 0)$. Figure 13 shows the comparative imaging results. In Figure 13(b), sparse ratio of receive array is set to 0.375 , and sampling rate is 0.4 which means the data is 0.15 times of original sample points. As shown in Figure 13, resolution is improved by 8 times in range and the range ambiguity suppression is better.

\section{Conclusion}

A MIMO SAR imaging based on compressed sensing is proposed in this paper to reduce the amount of data and suppress the range ambiguity. Random phase orthogonal waveform is designed for MIMO SAR based on compressed 


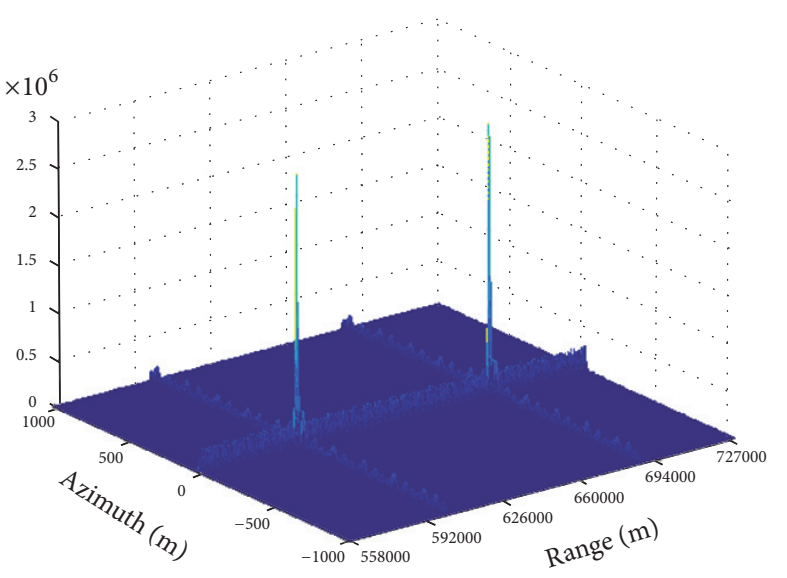

(a) Imaging of HRWS SAR



(b) Imaging of MIMO SAR based on CS

FIGURE 13: Imaging of HRWS SAR and MIMO SAR based on CS.

sensing. Using DBF technology in transmit and receive, the echoes of RPOW enable range resolution improved and the range ambiguity is suppressed by zero-point technology based on CS. However, due to the characteristics of signal recovery based $\mathrm{CS}$, it is required that the signal be sparse on a projection basis. In the case of small signal, the sparse coefficient is submerged in the noise, and the recovery of signal can be distorted. Therefore, this method has a certain scope of application.

\section{Competing Interests}

The authors declare that there is no conflict of interests regarding the publication of this paper.

\section{Acknowledgments}

This work was supported by the Chinese Defense Advance Research Program of Science and Technology of China (no. 403040101).

\section{References}

[1] W.-Q. Wang and J. Cai, "MIMO SAR using chirp diverse waveform for wide-swath remote sensing," IEEE Transactions on Aerospace \& Electronic Systems, vol. 48, no. 4, pp. 3171-3185, 2012.

[2] H. L. V. Trees, "Detection, estimation, and modulation theory, part IV," Optimum Array Processing, vol. 1, no. 2, pp. 660-678, 2003.

[3] G. Ruvio, R. Solimene, A. Cuccaro, D. Gaetano, J. E. Browne, and M. J. Ammann, "Breast cancer detection using interferometric MUSIC: experimental and numerical assessment," Medical Physics, vol. 41, no. 10, 2014.

[4] G. D. Callaghan and I. D. Longstaff, "Wide-swath space-borne SAR using a quad-element array," IEE Proceedings: Radar, Sonar and Navigation, vol. 146, no. 3, pp. 159-165, 1999.

[5] N. Gebert, G. Krieger, and M. A. Moreira, "Digital beamforming on receive: techniques and optimization strategies for highresolution wide-swath SAR imaging," IEEE Transactions on
Aerospace and Electronic Systems, vol. 45, no. 2, pp. 564-592, 2009.

[6] D. Cerutti-Maori, I. Sikaneta, J. Klare, and C. H. Gierull, "MIMO SAR processing for multichannel high-resolution wide-swath radars," IEEE Transactions on Geoscience and Remote Sensing, vol. 52, no. 8, pp. 5034-5055, 2014.

[7] X. Zhang, P. Huang, and X. Li, "2D SAR imaging scheme based on compressive sensing," Electronics Letters, vol. 50, no. 2, pp. 114-116, 2014.

[8] F.-F. Gu, Q. Zhang, L. Chi, Y.-A. Chen, and S. Li, "A novel motion compensating method for MIMO-SAR imaging based on compressed sensing," IEEE Sensors Journal, vol. 15, no. 4, pp. 2157-2165, 2015.

[9] J. Chang, W. Zhang, S. Zhang, and J. Li, "A novel SAR imaging algorithm based on compressed sensing," in Proceedings of the IEEE CIE International Conference on Radar, pp. 1467-1470, IEEE, Chengdu, China, October 2011.

[10] J. Guo, J. Zhang, K. Yang, B. Zhang, W. Hong, and Y. Wu, "Information capacity and sampling ratios for compressed sensing-based SAR imaging," IEEE Geoscience and Remote Sensing Letters, vol. 12, no. 4, pp. 900-904, 2015.

[11] J. Fang, Z. Xu, B. Zhang, W. Hong, and Y. Wu, "Fast compressed sensing sar imaging based on approximated observation," IEEE Journal of Selected Topics in Applied Earth Observations and Remote Sensing, vol. 7, no. 1, pp. 352-363, 2014.

[12] W.-Q. Wang, "Space-time coding MIMO-OFDM SAR for high-resolution imaging," IEEE Transactions on Geoscience and Remote Sensing, vol. 49, no. 8, pp. 3094-3104, 2011.

[13] W.-Q. Wang, "Large time-bandwidth product MIMO radar waveform design based on chirp rate diversity," IEEE Sensors Journal, vol. 15, no. 2, pp. 1027-1034, 2015.

[14] W. Mehany, L. Jiao, and K. Hussien, "Orthogonal discrete frequency-coding waveform design based on modified genetic algorithm for MIMO-SAR," in Proceedings of the 9th IEEE Conference on Industrial Electronics and Applications (ICIEA '14), pp. 1082-1086, Hangzhou, China, June 2014.

[15] F. Liu, S. Mu, W. Lv, W. Li, T. Ge, and L. Li, "LFM hybridcoding waveform design of MIMO SAR based on NSGAII," in Proceedings of the International Conference on Wireless Communications and Signal Processing (WCSP '15), Nanjing, China, October 2015. 
[16] R. Baraniuk, M. Davenport, R. DeVore, and M. Wakin, "A simple proof of the restricted isometry property for random matrices," Constructive Approximation, vol. 28, no. 3, pp. 253263, 2008.

[17] Y. Zhao, Y. H. Hu, and H. Wang, "Enhanced random equivalent sampling based on compressed sensing," IEEE Transactions on Instrumentation and Measurement, vol. 61, no. 3, pp. 579-586, 2012.

[18] C. Ekanadham, D. Tranchina, and E. P. Simoncelli, "Recovery of sparse translation-invariant signals with continuous basis pursuit," IEEE Transactions on Signal Processing, vol. 59, no. 10, pp. 4735-4744, 2011.

[19] S. S. Chen, D. L. Donoho, and M. A. Saunders, "Atomic decomposition by basis pursuit," SIAM Review, vol. 43, no. 1, pp. 129-159, 2001.

[20] L.-H. Chang and J.-Y. Wu, "An improved RIP-based performance guarantee for sparse signal recovery via orthogonal matching pursuit," IEEE Transactions on Information Theory, vol. 60, no. 9, pp. 5702-5715, 2014.

[21] J. A. Tropp and A. C. Gilbert, "Signal recovery from random measurements via orthogonal matching pursuit," Institute of Electrical and Electronics Engineers. Transactions on Information Theory, vol. 53, no. 12, pp. 4655-4666, 2007.

[22] J. Wang, W.-X. Sheng, Y.-B. Han, and X.-F. Ma, "Adaptive beamforming with compressed sensing for sparse receiving array," IEEE Transactions on Aerospace and Electronic Systems, vol. 50, no. 2, pp. 823-833, 2014. 


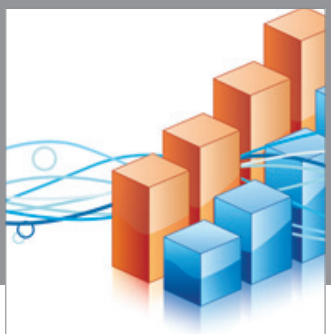

Advances in

Operations Research

vatem alat4

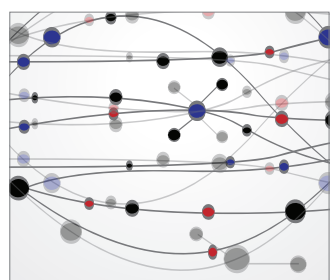

\section{The Scientific} World Journal
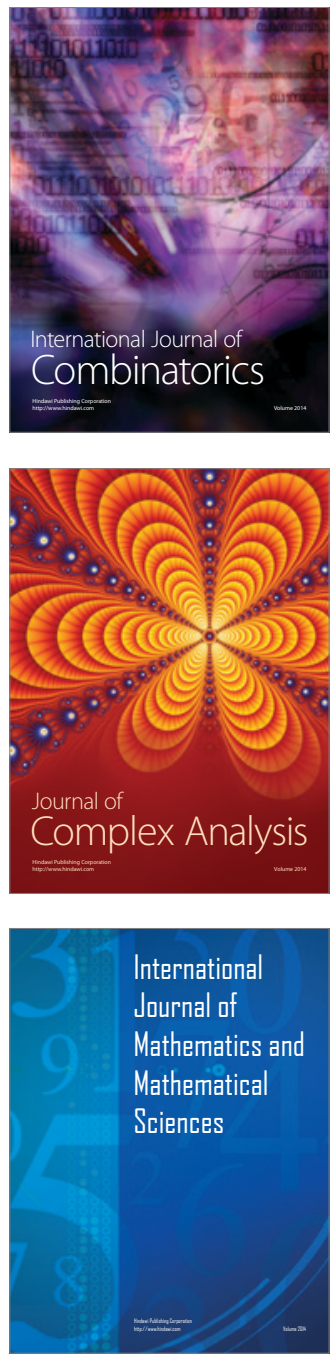
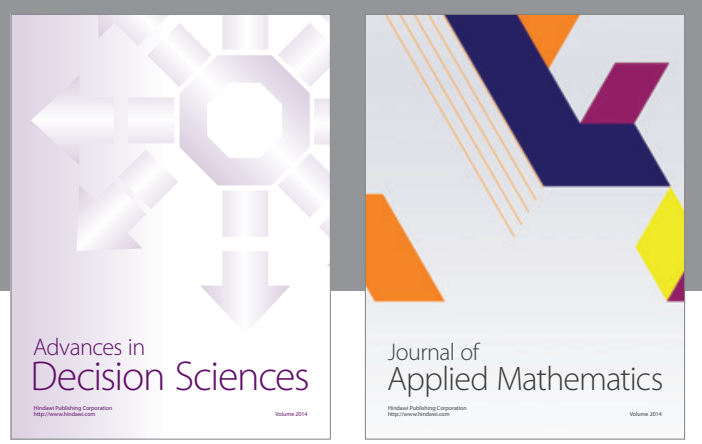

Algebra

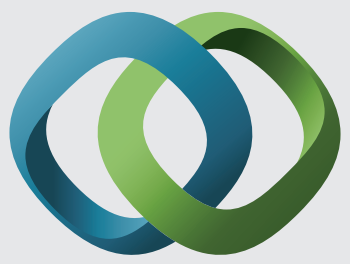

\section{Hindawi}

Submit your manuscripts at

https://www.hindawi.com
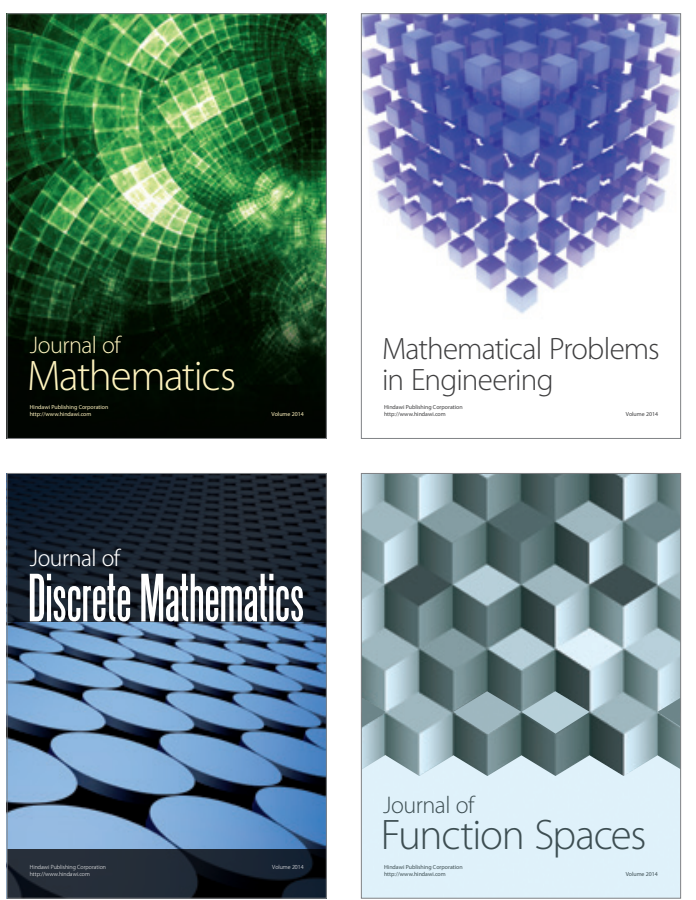

Mathematical Problems in Engineering
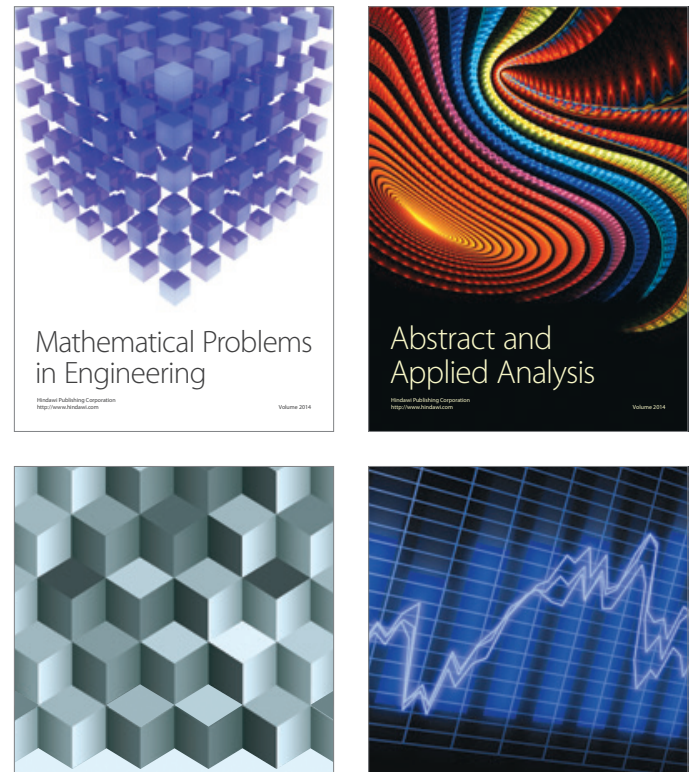

Journal of

Function Spaces

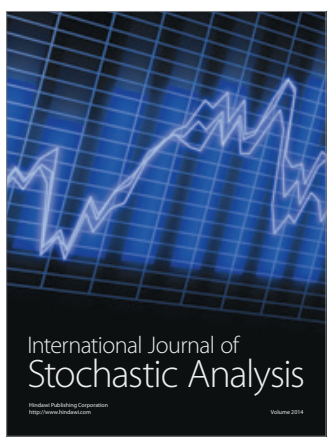

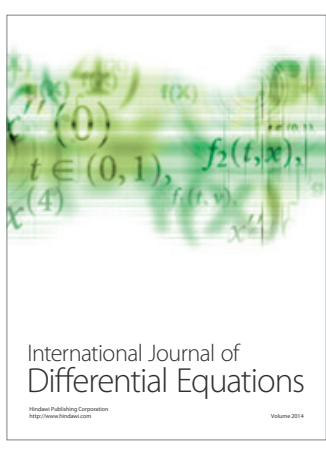
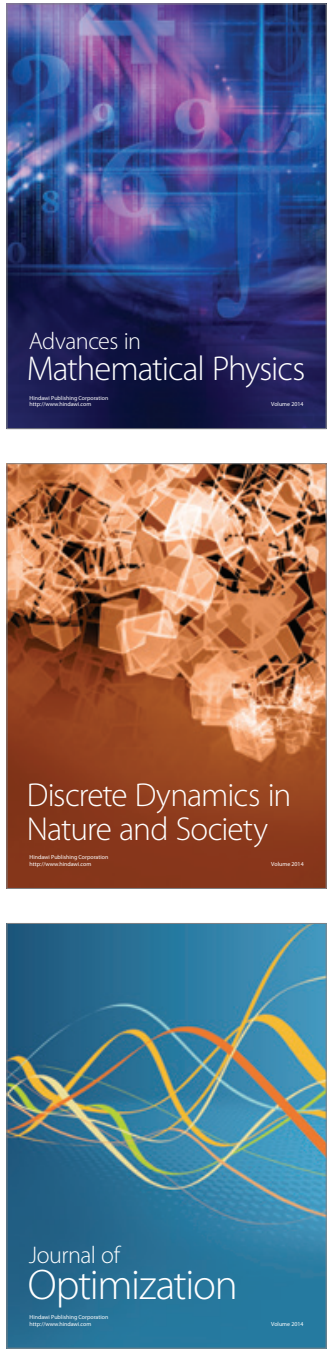